\title{
Dendritic Remodeling and Growth of Motoneurons during Metamorphosis of Drosophila melanogaster
}

\author{
Christos Consoulas, Linda L. Restifo, and Richard B. Levine \\ Arizona Research Laboratories, Division of Neurobiology, University of Arizona, Tucson, Arizona 85721
}

Insect motoneurons display dramatic dendritic plasticity during metamorphosis. Many larval motoneurons survive to adulthood but undergo dendritic regression and outgrowth as they are incorporated into developing circuits. This study explores the dendritic remodeling and development of Drosophila motoneurons MN1-MN5, which innervate indirect flight muscles of the adult. MN1-MN5 are persistent larval neurons exhibiting two distinct metamorphic histories. MN1-MN4 are born in the embryo, innervate larval muscles, and undergo dendritic regression and regrowth during metamorphosis. MN5, which was identified through a combination of intracellular dye injection and retrograde staining at all stages, is also born embryonically but remains developmentally arrested until the onset of metamorphosis. In the larva, MN5 lacks dendrites, and its axon stops in the mesothoracic nerve without innervating a target muscle. It is dye coupled to the peripherally synapsing interneuron, which will become part of the giant fiber escape circuit of the adult fly. During pupal development, MN5 undergoes de novo dendritic growth and extension of its axon to innervate the developing target muscle. Its unique developmental history and identifiability make MN5 well suited for the study of dendritic growth using genetic and neurophysiological approaches.

Key words: insect; motor neuron; dendrite; dendritic plasticity; CNS; spine; indirect flight muscles
The formation of precise dendritic shapes during development has important consequences for the integration and transmission of information in the nervous system. Modifications in dendritic architecture in the mature nervous system can either refine or impair the function of neural circuits (Bottjer and Arnold, 1997; Woolley, 1998; McEwen, 1999; Cameron and Nuñez-Abades, 2000; Swann et al., 2000; Cline, 2001; Poirazi and Mel, 2001). Dendrites of CNS neurons of holometabolous insects are remodeled during metamorphosis to allow the production of stagespecific behavior (Levine et al., 1995). In the moth Manduca sexta, many larval motoneurons survive to function as elements of the adult CNS. Persistent motoneurons undergo severe dendritic pruning during the larval-to-pupal transition, followed by dendritic growth during adult development (Levine and Truman, 1982, 1985; Thorn and Truman, 1989; Weeks and ErnstUtzschneider, 1989; Kent and Levine, 1993; Consoulas et al., 2000; Duch and Levine, 2000). Motoneuron dendritic remodeling is regulated by the steroid hormone 20-hydroxyecdysone (for review, see Levine and Weeks, 1990; Weeks and Levine, 1990; Levine et al., 1995), but the molecular mechanisms remain unknown.

Another holometabolous insect, Drosophila melanogaster, undergoes equally spectacular behavioral changes but also offers molecular genetic tools for exploring the signals and transduction pathways that regulate dendritic shape and plasticity (Gao et al., 1999). Little is known, however, about dendritic remodeling of

\footnotetext{
Received Oct. 9, 2001; revised Feb. 1, 2002; accepted Feb. 11, 2002.

This work was supported by National Institutes of Health Grant NS28495. We thank Dr. C. Duch for comments on this manuscript, C. Michel for preparing fly food, and Dr. R. Kraft for valuable advice on fly genetics throughout the course of this study. We also thank Drs. R. Murphey and K. Ito for fly stocks.

Correspondence should be addressed to Dr. Christos Consoulas at his present address: National University of Athens, Medical School, Laboratory of Experimental Physiology, Micras Asias 75, 11527 Athens, Greece. E-mail: cconsoul@med. uoa.gr.

Copyright (C) 2002 Society for Neuroscience $\quad 0270-6474 / 02 / 224906-12 \$ 15.00 / 0$
}

identified neurons in Drosophila because of the experimental restrictions imposed by the small size of the animal and its CNS. Thus, the goal of this study was to establish a model system that will allow high-resolution analysis of dendritic structure of individually identified central neurons of Drosophila.

Here, by using intracellular staining and axonal tracing techniques, we determined the developmental histories of motoneurons MN1-MN5, which innervate the dorsolongitudinal indirect flight muscles (DLM) in the adult fly (Coggshall, 1978; King and Wyman, 1980; Costello and Wyman, 1986; Ikeda and Koenig, 1988; Sun and Wyman, 1997). The DLMs develop from three larval dorsal mesothoracic body wall muscles, which, unlike other thoracic muscles, escape histolysis during the larval-to-pupal transition (Shatoury, 1956; Costello and Wyman, 1986; Fernandes et al., 1991). In the prepupa and early pupa, the motor nerve that innervates the dorsal larval mesothoracic muscles remains in contact with the larval templates and, in later pupal stages, grows over the developing DLMs (Fernandes and Vijayraghavan, 1993). The continuous nerve-muscle association during metamorphosis suggests that DLMs are innervated by persistent larval neurons, but MN1-MN5 had never been identified at larval or prepupal stages. This was a prerequisite for determining their origins and developmental histories.

\section{MATERIALS AND METHODS}

Drosophila strains and culture. Canton-S was the wild-type laboratory strain as in previous studies of CNS metamorphosis (Restifo and White, 1991; Restifo et al., 1995; Restifo and Hauglum, 1998). Experimental animals were cultured on standard corn flour-yeast-agar medium (Elgin and Miller, 1978) at $25^{\circ} \mathrm{C}, 50-80 \%$ relative humidity. 5-Bromo-2deoxyuridine (BrdU) (Sigma, St. Louis, MO) was used to label cells undergoing DNA synthesis. BrdU was added to the culture medium at a concentration of $0.1 \mathrm{mg} \mathrm{BrdU} / \mathrm{ml}$ (Truman and Bate, 1988). Animals were continuously exposed to BrdU throughout larval development. Wandering third instar larvae were dissected and processed for BrdU immunocytochemistry as described previously (Consoulas and Levine, 1997). 
White prepupae ( $\mathrm{P} 1$, according to the staging criteria of Bainbridge and Bownes, 1981) were selected from culture vials, placed on moistened ashless filter paper (catalog \#42; Whatman, Maidstone, UK) in glass dishes, and allowed to continue developing at $25^{\circ} \mathrm{C}$ in a humid chamber for varying numbers of hours after puparium formation (hr APF). Under these conditions, pupation takes places at $12 \mathrm{hr}$ APF on average, with a range of 11-13 hr APF (L. L. Restifo and W. Hauglum, unpublished observation). Eclosion takes place at $\sim 100 \mathrm{hr}$ APF.

A number of $P[G A L 4]$ enhancer-detector transgenes (Brand and Perrimon, 1993) were used to label CNS landmarks. GAL4 expression was localized by crossing to flies homozygous for a second-chromosome insertion of the reporter construct, $P[U A S-G F P$.S65T], encoding mutant green fluorescent protein (GFP) (Cubitt et al., 1995). The GFP construct and transgenic line were made by B. Dickson (University of Vienna, Vienna, Austria; FlyBase accession number FBrf0086268), and the stock was provided by K. Ito (National Institute for Basic Biology, Myodaiji, Okazaki, Japan).

$P[G A L 4] 103.3$ is a second-chromosome insertion (cytogenetic map location 48C3-5), identified in a screen by the Nambu and Murphey laboratories (University of Massachusetts-Amherst, Amherst, MA), that drives expression in adult indirect flight muscle motoneurons (R. Murphey, personal communication). DLM motoneuron expression does not begin until $35 \mathrm{hr}$ APF (data not shown).

Retrograde and anterograde nerve filling technique. The animals were anesthetized by chilling on ice for 3-5 min. Pupae were removed from their puparia before dissection. Animals were pinned with dorsal side up on Sylgard-coated (Dow Corning, Midland, MI) Petri dishes containing HL3 saline (Stewart et al., 1994). The heads of late pupae and adults were removed. Larvae, prepupae and early pupae (until $18 \mathrm{hr}$ APF) were dissected along the right dorsolateral region to leave the left hemisegments intact. For later pupal stages and the adult, the animals were dissected along the dorsal midline. The gut, salivary glands, fat bodies, and trachea were removed. For retrograde labeling of the motoneurons in the larva, prepupa, and early pupa, the specific branch of the nerve containing the motoneurons innervating the DLMs or their precursors was isolated together with its target muscles in a petroleum jelly pool to allow the uptake of dye [5\% w/v rhodamine-dextran at 3000 or 10,000 $\mathrm{kDa}$ in distilled water (Molecular Probes, Eugene, OR) or $3 \% \mathrm{w} / \mathrm{v}$ biocytin in distilled water (Sigma)]. For anterograde staining of the peripheral processes of the mesothoracic (T2) neurons, the whole CNS, with only the T2 nerve attached, was isolated in a petroleum jelly pool with the dyes (Consoulas et al., 1996). The rhodamine-dextran-labeled preparations were stored at $4^{\circ} \mathrm{C}$ for $3-7 \mathrm{hr}$ or at room temperature (RT) for a maximum of $3 \mathrm{hr}$ in the dark. The biocytin preparations were stored in $4^{\circ} \mathrm{C}$ for $5-10 \mathrm{hr}$. All preparations were fixed in $2.5 \%$ formaldehyde and $3 \%$ sucrose in PBS, $\mathrm{pH} 7.3$, for 2-5 hr at RT in the dark. The rhodaminedextran preparations were washed in PBS after fixation and mounted in $80 \%$ glycerol in PBS. Biocytin preparations were washed in PBS, $\mathrm{pH} 7.3$, and PBS-Tx (0.4\% Triton X-100 in PBS, $\mathrm{pH} 7.3)$ and then incubated in 1:2000 Cy3- or Cy5-conjugated Streptavidin (Jackson ImmunoResearch, West Grove, PA) in PBS-Tx for $2 \mathrm{hr}$ at RT in the dark. Preparations were mounted in $80 \%$ glycerol in PBS. After fixation (see above), filamentous actin within the muscles was labeled with $66 \mathrm{~nm}$ Oregon Green-phalloidin (Molecular Probes) in PBS for $30 \mathrm{~min}$ at RT (Consoulas and Levine, 1998).

Intracellular staining technique. Larvae, prepupae, and young pupae (up to $35 \mathrm{hr}$ APF) were prepared as described above. To identify MN5 and the $X$ neuron (see Results) the T2 nerve was first backfilled with 3000 $\mathrm{kDa}$ rhodamine-dextran. After dye infusion for 25-90 min at RT in the dark, the preparation was transferred to a different dish and pinned on a Sylgard platform to allow impalement. In pupae past $35 \mathrm{hr}$ APF and adults, it was possible to visually locate the MN5 cell body using transmitted light and differential interference contrast optics, based on its size and position in the CNS. In addition, in P103.3 pupae older than $35 \mathrm{hr}$ APF and adults, the cell body of MN5 was located by its GFP expression. An Olympus Optical (Tokyo, Japan) microscope (BX50WI) equipped with fluorescent optics and 10 and $60 \times$ water-immersion objectives was used. In larvae, prepupae, and early pupae up to $18 \mathrm{hr}$ APF, the CNS sheath was removed mechanically with a fine pair of forceps. For late pupal stages (past $60 \mathrm{hr}$ APF) and adults, the CNS sheath was treated with $1 \% \mathrm{w} / \mathrm{v}$ pronase (Sigma) in saline. For pupae between 18 and $60 \mathrm{hr}$ APF, the sheath was thin enough to allow penetration by microelectrodes without any mechanical or chemical treatment. Thin-walled borosilicate electrodes (resistance of 60-80 M $\Omega$ ) were used to impale the neurons. The tips of the electrodes were filled with either $3 \%$ Lucifer yellow
(Sigma) in $2 \mathrm{M}$ lithium chloride (from wandering larvae to $25 \mathrm{hr}$ APF) or $3 \% \mathrm{w} / \mathrm{v}$ Neurobiotin (Sigma) in $2 \mathrm{M}$ potassium acetate. The electrode shafts were filled with $2 \mathrm{M}$ lithium chloride (Lucifer yellow electrodes) or $2 \mathrm{~m}$ potassium acetate (Neurobiotin electrodes). An air bubble was left between the tip and the shaft to prevent dye dilution. After intracellular penetration, Lucifer yellow was injected iontophoretically by applying 0.1-1.0 nA hyperpolarizing current for $10 \mathrm{~min}$ to $1 \mathrm{hr}$. Neurobiotin was injected by applying depolarizing current pulses of $1 \mathrm{nA}$ and $400 \mathrm{msec}$ duration with a frequency of $1 \mathrm{~Hz}$ for $5-15 \mathrm{~min}$. Subsequently, the preparations were fixed and washed in PBS. Neurobiotin preparations were processed as above. Preparations were mounted in $80 \%$ glycerol in PBS.

Confocal microscopy. Digital images were captured on a Nikon (Tokyo, Japan) PCM 2000 laser-scanning confocal microscope using Simple PCI (Compix, Tualatin, OR) image acquisition software. Preparations were scanned with helium-neon laser lines with excitation maxima at $543 \mathrm{~nm}$ (Cy3) and $650 \mathrm{~nm}$ (Cy5) using long-pass filters at 565 and $650 \mathrm{~nm}$, respectively, and with an argon laser line with an excitation maximum at $488 \mathrm{~nm}$ (GFP, fluorescein, and OR Green-phalloidin) using a bandpass filter at $500-530 \mathrm{~nm}$. Images were assembled using Corel Draw 8 software (Corel, Ottawa, Ontario).

\section{RESULTS}

\section{Comparison of motoneurons in the larva and adult}

Our ability to determine the developmental histories of the DLM motoneurons relied on a combination of intracellular staining and diffusion of dyes through cut motor axons (retrograde or anterograde staining). Whereas previous studies relied on HRP injection into the target muscles to identify the adult flight motoneurons (Ikeda and Koenig, 1988; Trimarchi and Schneiderman, 1994a), this approach would not suffice for our studies of earlier developmental stages in which the targets were unknown. Furthermore, as will be shown, previous retrograde staining facilitated greatly our subsequent ability to record intracellularly from MN5 of early developmental stages. Initial experiments tested the reliability of the staining technique by confirming the identity of the adult DLM motoneurons. Anterograde (toward the periphery) staining of the posterior branch of the dorsal mesothoracic nerve (PDMN) (Power, 1948) in the adult revealed that its distalmost branch is purely motor, containing the axons of the neurons innervating the six DLM fibers (Fig. 1 $A$ ). Retrograde staining of the distalmost PDMN branch in the adult revealed the position and dendritic architecture of five motoneurons (MN1MN5) (Fig. 1B,C) and of one ventral unpaired median neuron (VUM) (Fig. 1C) in the adult CNS. The cell bodies of MN1MN4 are located ventrally in the prothoracic neuromere (T1) ipsilateral to the target muscle (Fig. $1 C$ ). The cell body of MN5 is located dorsally in the mesothoracic (T2) neuromere on the contralateral side (Fig. $1 B$ ). The cell body of the VUM neuron is located posteriorly in the T2 neuromere at the ventral midline (Fig. 1C, VUM). The elaborate dendritic fields of MN1-MN5 are located dorsally on both ipsilateral and contralateral sides of the mesothoracic neuromere (Fig. 1B).

In the larva, anterograde nerve fills of the mesothoracic nerve (T2) revealed that the dorsal muscles are innervated by the distalmost branch of the intersegmental nerve (ISN) (Fig. 1D) (Fernandes and Vijayraghavan, 1993). Six large axonal profiles and usually a thin seventh axon, probably belonging to the VUM neuron, were visible in this nerve branch (Fig. 1E, inset). Retrograde staining of the same nerve branch revealed seven neurons in the larval CNS (Fig. $1 F, G$ ). Two of the neurons had cell bodies located dorsally, close to the midline in the ipsilateral mesothoracic neuromere [ipsilateral dorsal neurons a (anterior) (IDa) and p (posterior) (IDp)] (Fig. 1F). The cell bodies of four neurons were located ventrally, in the ipsilateral prothoracic neuromere 

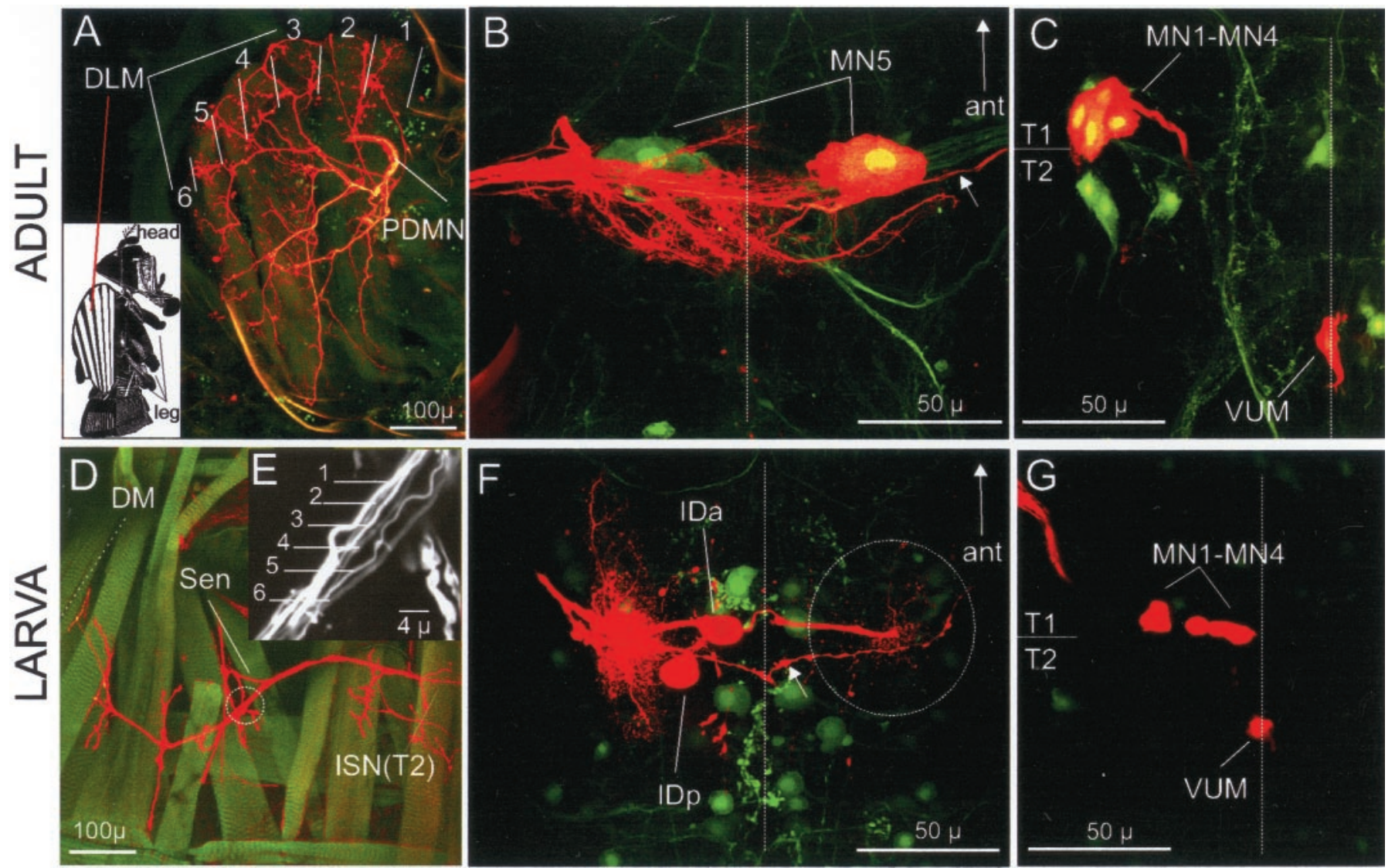

Figure 1. Innervation of the dorsal thoracic muscles in the larva and of the DLMs in the adult. All panels are projections of confocal optical sections $(z=0.6 \mu \mathrm{m}$ in $B, C, E-G ; z=1.5 \mu \mathrm{m}$ in $A, D)$ taken from adult $(A-C)$ and larval $(D-G)$ stages of P103.3/UAS-GFP animals. In all panels, anterior is to the top. Dotted lines in $B, C, F$, and $G$ indicate the CNS midline. The GFP reporter ( green in $B, C, F, G$ ) labels MN1-MN5 in the adult, among other neurons. The motoneurons innervating the dorsal muscles do not express GFP in the larva, although many other neurons do, providing a useful marker for the CNS midline. A, Anterograde staining (red) of the PDMN nerve revealed the innervation pattern of the six DLM fibers (see numbers) in the adult $B, C$, Retrograde staining (red) of the distalmost branch of PDMN, which specifically innervates the DLMs, revealed MN5 ( $B$, dorsal view), MN1-MN4, and the VUM neuron ( $C$, ventral view) in the adult CNS. Arrow in $B$ indicates the axon of the VUM neurons exiting the contralateral mesothoracic nerve. $D$, Anterograde staining of the T2 nerve revealed the innervation pattern of the larval mesothoracic muscles. The encircled area indicates the distalmost branch of the ISN just past the exit of the sensory (sen) branch. $E$, High-magnification view of the encircled area in $D$ showing the six motor axons innervating the dorsal muscles exclusively. $F, G$, Retrograde staining of the distalmost ISN nerve revealed two ipsilateral dorsal neurons (IDa and IDp) $(F$, dorsal view) and four ventral ipsilateral neurons (MN1-MN4), along with a VUM neuron ( $G$, ventral view) innervating the larval mesothoracic dorsal muscles. Note in $F$ the contralateral dendrites (dotted circle) and the absence of a contralateral cell body. Arrow in $F$ indicates the axon of the VUM neurons exiting the contralateral mesothoracic nerve. The peripheral nerves in $A, D$, and $E$ were stained anterogradely with rhodamine-dextran $3 \mathrm{kDa}$ $(A)$ or biocytin $(D, E)$. The biocytin staining in $D$ and $E$ was revealed with Cy3-conjugated Streptavidin. In $A$ and $D$, the filamentous actin of the muscles was revealed with Oregon Green-phalloidin. In $B, C, F$, and $G$, the neurons in the CNS were retrogradely stained with rhodamine-dextran $3 \mathrm{kDa}$. $D M$, Dorsal midline $I S N(T 2)$, intersegmental nerve of the mesothoracic segment; ant, anterior.

(Fig. $1 G$ ). One neuron with a bifurcating axon, characteristic of unpaired neurons that project to both sides of the body, had its cell body in the ventral midline (Fig. $1 G, V U M$ ). The dendrites of all motoneurons were located ipsilaterally in the dorsal region, with the exception of one of the ventral motoneurons, which also had a dorsal dendritic field on the contralateral side (Fig. $1 F$, dotted circle). Judging from the position of the neuronal cell bodies in the larva, the four ventral-ipsilateral motoneurons may correspond to the adult MN1-MN4. Similarly, the larval VUM neuron may survive to the adult stage to innervate the DLMs.

Because no larval neuron with a contralateral cell body was found to innervate the dorsal muscles, the origin of the adult MN5 was not obvious. One hypothesis is that MN5 is born during larval life. Alternatively, the IDa or IDp neuron may migrate during metamorphosis to the contralateral side of the CNS to become MN5, or MN5 may be a larval motoneuron innervating muscles other than those in the dorsal body wall. Finally, MN5 may be present in the larva but have no muscle target. The following experiments resolved this issue.

\section{Changes in the larval population of motoneurons during metamorphosis}

The retrograde labeling technique provided novel information about changes in the population of larval motoneurons during the early stages of metamorphosis. The number of primary ISN nerve branches did not change during metamorphosis. As in the larval stage, the distalmost ISN branch innervated the DLM templates in the prepupa and the developing DLMs in the pupa (our unpublished observations) (Fernandes and Vijayraghavan, 1993). Retrograde fills from this branch in the late larval and very early prepupal stages ( $0-2 \mathrm{hr}$ APF) revealed no changes from the larval number, position, and dendritic pattern of the efferent neurons. However, regression of dendrites began by $2 \mathrm{hr}$ APF and was completed by $4 \mathrm{hr}$ APF (Fig. 2B-D). Within the next two hours 

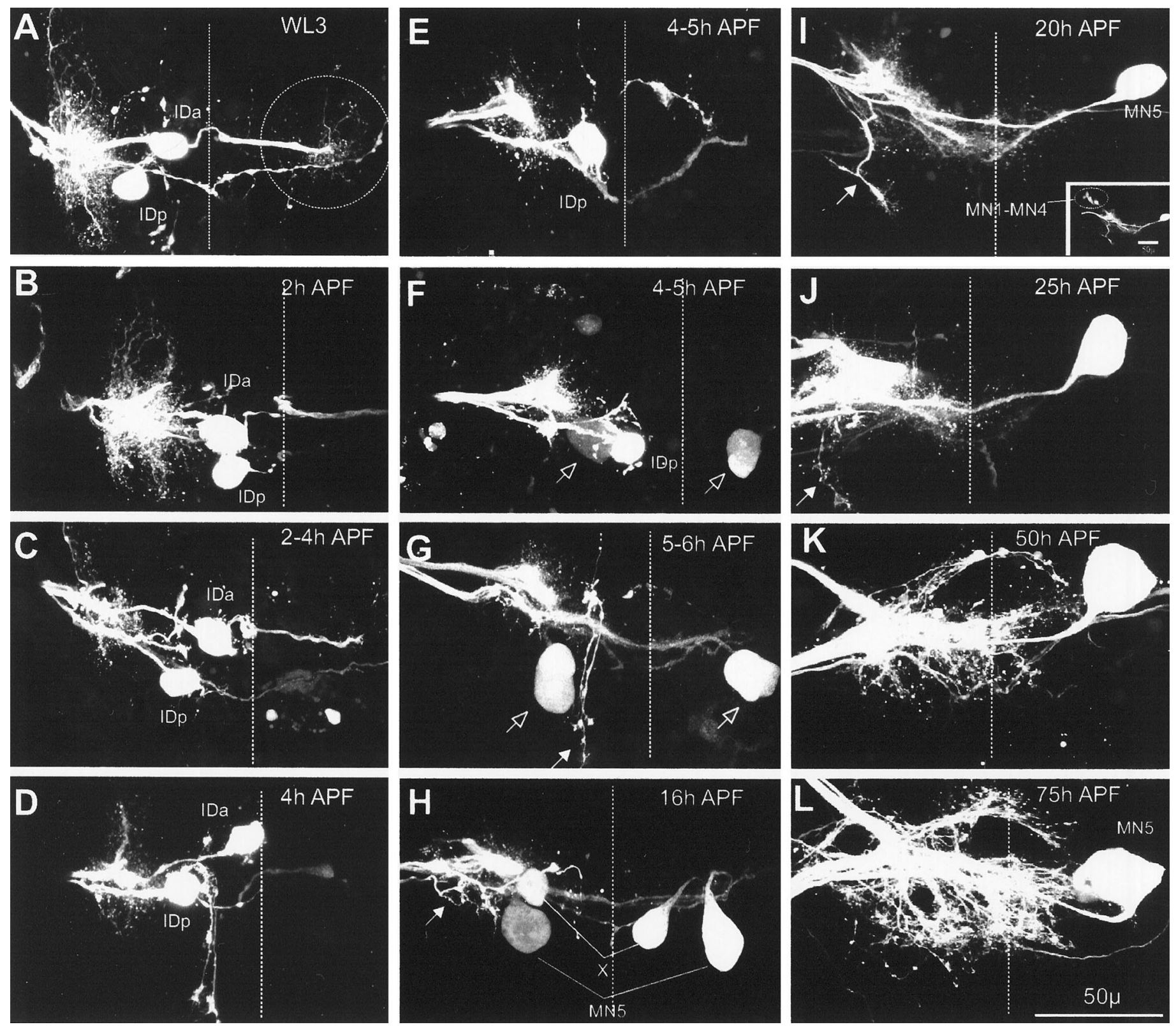

Figure 2. Metamorphic remodeling of thoracic motoneurons. $A-J$, Projections of confocal optical sections $(z=0.6 \mu \mathrm{m})$ from $P 103.3 / U A S$-GFP animals at stages indicated in the top right of each panel. The branch of the intersegmental nerve (to the left of each image) that innervates the developing DLMs exclusively was stained retrogradely with rhodamine-dextran $3 \mathrm{kDa}$. The rhodamine signal is shown; the GFP-labeled cells (data not shown) were used to identify the midline (dotted line) and segment borders. All of the images, except inset in $I$, are dorsal views of the CNS, showing the dorsal cell bodies and the axons and dendritic arbors of all the motoneurons. The MN1-MN4 ventral cell bodies were present in all of the preparations, but they are shown only in inset in $I$. $A-C$, The larval dendrites $(A)$ (see Fig. $1 F$ ) begin to undergo retraction within 2 hr of pupariation $(B, C)$. The encircled area indicates the contralateral dendrites that are almost completely retracted by $4 \mathrm{hr}$ APF. $D-G$, IDa and IDp neurons disappear sequentially within the next 2 hr (4-6 hr APF). F, $G$, At the same time, two pairs of closely associated dorsal cells become apparent (open arrows in $F, G$ ). These pairs of neuronal cell bodies (MN5 and X) become separated by $16 \mathrm{hr}$ APF $(H)$. I, By $20 \mathrm{hr}$ APF, only one contralateral cell (MN5) appears in backfills in the dorsal region of the CNS. At the same time, the MN1-MN5 dendrites begin to expand. $J-L$, Dendrite sprouting and growth continues during the next stages. The dendritic pattern becomes adult-like by $75 \mathrm{hr}$ APF (compare with Fig. $1 B$ ). Arrows in $H-J$ indicate a sensory branch that was stained accidentally. Note that the MN5 cell body increases considerably in size (see also Fig. 6).

(4-6 hr APF) all larval thoracic muscles degenerated, with the exception of the three DLM template fibers. The four ipsilateralventral motoneurons and the VUM neuron were still present in nerve fills, but the two dorsal ipsilateral motoneurons (IDa and IDp) disappeared sequentially, with IDa first, followed by IDp (Fig. 2D-F). These two larval neurons were not present in whole-T2 nerve fills, as well, suggesting that they degenerate.

Before the IDp motoneuron disappeared from retrograde fills, two pairs of neurons became apparent in ipsilateral and contralateral positions, respectively, on the dorsal surface of the mesothoracic neuromere (Fig. $2 F, G$ ). The same two pairs of cells were stained in fills of the left or right ISN branch. The contralateral pair was always more heavily labeled than the ipsilateral pair. All retrograde fills described above were performed with $3 \mathrm{kDa}$ rhodamine-dextran, which is small enough to cross gap junctions. Using dyes of different molecular weights, we demonstrated that 

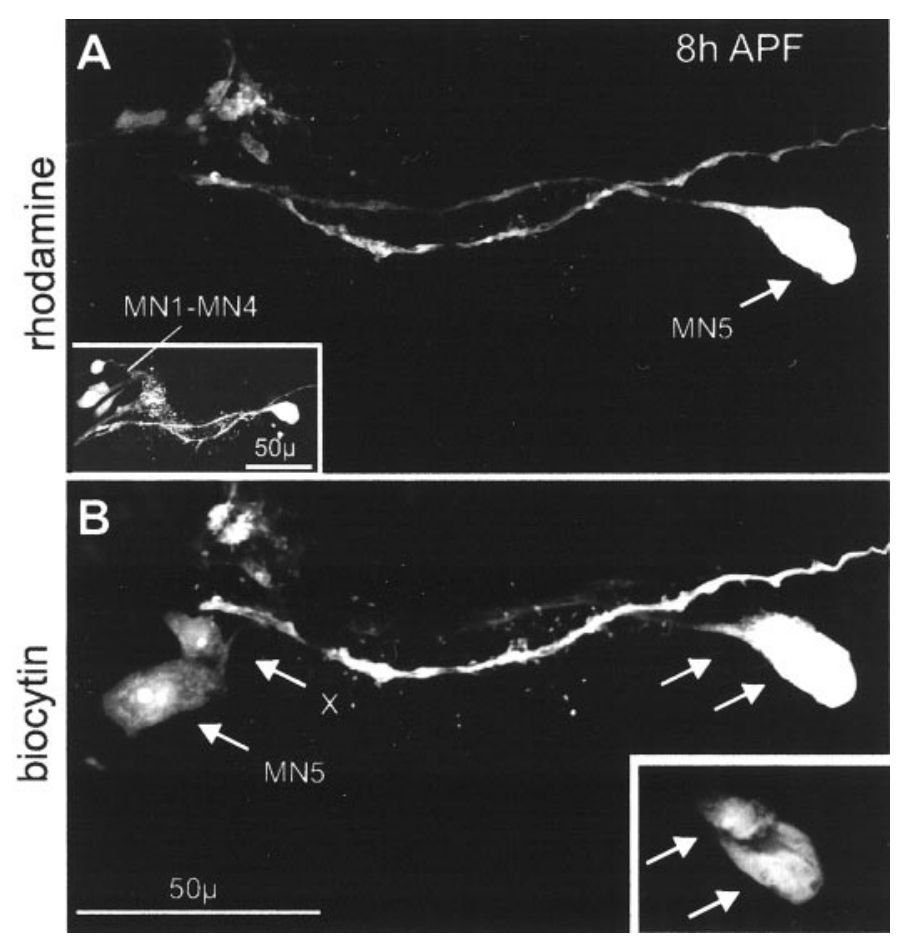

Figure 3. MN5 and X neuron are dye coupled in the prepupa and early pupa. $A, B$, Projections of confocal optical sections of an $8 \mathrm{hr}$ APF Canton-S preparation show the dorsal region. The distalmost branch of the left ISN was filled with a mixture of $10 \mathrm{kDa}$ rhodamine-dextran and biocytin. The image in $A$ shows only the rhodamine fluorescence, and $B$ shows the biocytin revealed with Cy5-conjugated Streptavidin. Although biocytin diff used into both pairs of MN5 and X neurons ( $B$, arrows), only the contralateral (right) MN5 was labeled with $10 \mathrm{kDa}$ rhodaminedextran $(A)$. This indicates that the ipsilateral pair of neurons and the contralateral X neuron are dye coupled to the contralateral MN5. Inset in $A$ shows the same population at lower magnification. Note that the ventral cell bodies of MN1-MN4 are stained along with MN5 by the $10 \mathrm{kDa}$ rhodamine-dextran. Inset in $B$ is a single confocal optical section, showing the distinct cell bodies of the contralateral MN5 and X neuron, which are difficult to distinguish in the merged image above.

this staining pattern was the result of dye coupling among the four neurons. When $10 \mathrm{kDa}$ rhodamine-dextran, which does not cross gap junctions (Phelan et al., 1996), was used in combination with biocytin, the dyes colocalized only in a single neuron, the larger contralateral one, suggesting that only this neuron innervates the DLM templates on the side of the fill (Fig. 3A,B). This neuron was identified as MN5, with further evidence provided below.

By $16 \mathrm{hr}$ APF, the two cell bodies of each neuronal pair were clearly separated from each other, but all four were still dye coupled (Fig. 2H). By 18 hr APF, only one contralateral-dorsal neuron was present in fills of the same nerve branch, together with the four ipsilateral-ventral motoneurons and the VUM neuron. The position and the number of neurons did not change during the remaining pupal stages, suggesting that the four ventral-ipsilateral larval neurons are MN1-MN4 and that the contralateral-dorsal neuron is MN5. The inability to reveal the putative MN5 with retrograde staining from distal sites before 4-5 hr APF suggests that its axon first reaches the DLM template at this stage. The other contralateral neuron, which is indirectly labeled by retrograde filling of the distal nerve between 4-5 and $18 \mathrm{hr}$ APF, will be called X. The MN5 and X neurons are considered in detail in the next section below.

The retracted motoneuron dendritic arbors began to reexpand by 19-20 hr APF (Fig. 2I). The first phase of dendritic growth (19-25 hr APF) was highlighted by the presence of numerous fine processes, mainly in the ipsilateral mesothoracic neuromere (Fig. $2 I, J)$. By $50 \mathrm{hr}$ APF, numerous dendrites had grown perpendicular to their primary neurite. Some of the anterior branches had bent and crossed the CNS midline (Fig. $2 K$ ). By $75 \mathrm{hr}$ APF, the dendritic pattern had most of its adult features (Fig. $2 L$ ), but the growth of very high-order branches continued until adult emergence, which typically occurred at $\sim 100 \mathrm{hr}$.

\section{The developmental history of MN5}

The preceding experiments brought into question the origin of MN5. The larval MN5 may innervate a muscle that does not belong to the dorsal group. This would explain why MN5 was not stained in retrograde fills of the distalmost branch of the larval ISN. To examine whether MN5 innervates targets different from the larval dorsal muscles, every individual subbranch or group of branches of the larval T2 nerve was filled. Two cell bodies belonging to the ventral longitudinal muscle motoneurons (VLmns) were located contralaterally, close to the midline. No other neurons with contralateral cell bodies were revealed (data not shown). However, when the entire larval T2 nerve was filled from a very proximal location just outside the CNS, a pair of cells, reminiscent of the MN5 and X neurons, were faintly labeled, in addition to the heavily labeled IDa, IDp, and VLmns (Fig. 4A). These neurons had not been stained in more distal fills from individual branches of the larval T2 nerve, suggesting either that their axons stop in the main T2 nerve or that they are dye coupled to other mesothoracic neuron(s) that have an axon in the proximal nerve.

By transecting at different points along the larval mesothoracic nerve, it was determined that backfills from a location just proximal to the point at which T2 splits into the segmental nerve (SN) and the ISN (Fig. 4C, arrow) consistently revealed the contralateral pair of neurons and the homologous ipsilateral pair (Fig. 4B). As in the late prepupa and early pupa, the ipsilateral cell bodies were always less well stained and were usually obscured by the larval dendrites of other neurons. In contrast, only a single, contralateral cell was revealed in backfills with $10 \mathrm{kDa}$ rhodamine-dextran (data not shown), suggesting that the other three cells were dye coupled to the fourth cell or to other neurons with axons in the nerve.

None of the neurons that were labeled by retrograde fills of the entire T2 nerve were derived from the neuroblast divisions that occur during the larval instars. Females were allowed to lay eggs in food that contained the thymidine analog BrdU. This resulted in incorporation of BrdU by those cells that underwent DNA synthesis during the larval stages. Biocytin fills of the entire proximal T2 nerve of wandering third instar larvae were performed. BrdU incorporation was evident in the progeny of neuroblasts that divided in the larva (Truman and Bate, 1988) but not in the biocytin-labeled neurons (Fig. 4A).

Intracellular dye injection revealed the morphology of the putative MN5 and the X neuron in the larva. First, to reveal the two right-hand cell bodies, the left T2 nerve was cut close to the CNS and filled with $3 \mathrm{kDa}$ rhodamine-dextran (Fig. $5 A$ ). One of the labeled cell bodies was then impaled under visual control with a microelectrode containing Lucifer yellow (see Materials and Methods) (Fig. 5A). Within 20-45 sec of the onset of intracellular injection, the dye had diffused into the other three cell bodies (Fig. 5B), suggesting the presence of gap junctions among the four neurons. The four cell bodies were associated with only two axons running in close apposition; only one member of the pair on each 

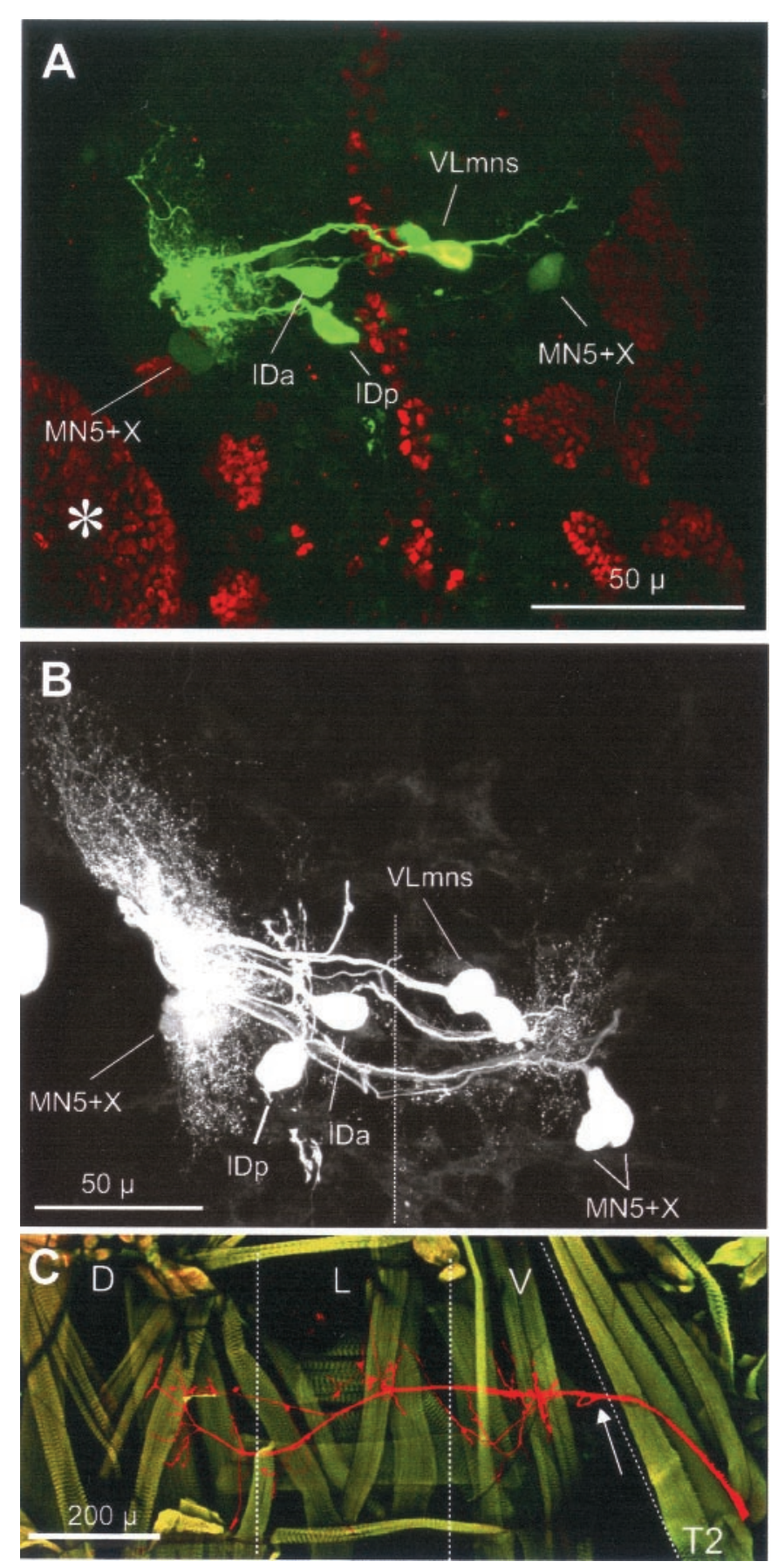

Figure 4. Mesothoracic motoneurons are embryonic in origin. All panels are projections of confocal optical sections $(z=0.5 \mu \mathrm{m})$ from Canton-S animals. $A$, Dorsal region of the thoracic neuromeres of a wandering third instar larva that had fed throughout its life on culture medium with BrdU. The entire mesothoracic nerve on the left was filled with biocytin, and the staining was revealed with Cy5-conjugated Streptavidin (green). The BrdU, which was incorporated by the nuclei of cells undergoing DNA synthesis during larval life, was revealed with a monoclonal antibody conjugated to $\mathrm{Cy} 3$ (red). The biocytin-filled mesothoracic neurons did not incorporate $\mathrm{BrdU}$, suggesting that they have an embryonic origin. Note the presence of MN5 and X neurons lightly stained with biocytin bilaterally. Asterisk indicates the mesothoracic leg imaginal disc, whose nuclei had incorporated BrdU. B, Dorsal region of the larval CNS. The mesothoracic nerve on the left was filled with $3 \mathrm{kDa}$ rhodamine-dextran (red) at a level proximal to the first branch point (arrow in $C$ ). Dotted line represents the midline. In addition to IDa, IDp, and VLmns, two pairs of cells (MN5 and X) are apparent. Note that the ipsilateral pair (left) is obscured by the dense dendritic arbors. $C$, Body wall preparation of the side had an efferent axon in the larva. These neurons lacked dendritic arbors (Fig. 5C). Conspicuous swellings were observed in the axons (Fig. 5C, arrowhead), which may indicate contact points between the two axons.

An additional step confirmed that the larval axon of the putative MN5 is very short, terminates without innervating a muscle, and does not project to a branch of the T2 nerve that was missed in our retrograde fills. After filling the left T2 nerve with $3 \mathrm{kDa}$ rhodamine-dextran, the larger of the two right-hand cell bodies was injected with Lucifer yellow. The two left-hand cell bodies were stained as a consequence of dye transfer, and the larger of the two was then impaled under visual control. Intracellular injection with Lucifer yellow for 30-45 min allowed the dye to fill its intact axon entirely (Fig. 5D,E). This experimental approach confirmed unambiguously that the larval MN5 axon stops within the T2 nerve just before its first branching point (Fig. $5 F$ ). In other words, in the mature larva, MN5 is present but incompletely differentiated. It has no dendrites and a short axon that does not innervate a muscle.

The retrograde labeling experiments confirmed that MN5 first reaches the DLM template fibers between 4 and $5 \mathrm{hr}$ APF (Fig. $2 F)$. Its axon must, therefore, extend toward the dorsal region of the mesothoracic segment during the interval between wandering third larval instar and $6 \mathrm{hr}$ APF. Indeed, prolonged intracellular injection of Lucifer yellow into the left MN5 during the 4-6 hr APF interval confirmed that its axon had almost reached the dorsal region of the right mesothoracic hemisegment (Fig. 5G-I).

In the same preparation, the left $\mathrm{X}$ neuron was also stained as a result of dye coupling (Fig. $5 G$ ), but its axon did not exit the CNS. In contrast to the larval stage, in prepupal and early pupal preparations (2-18 hr APF), Lucifer yellow diffusion to the contralateral pair of MN5 and X cell bodies became evident only after $1 \mathrm{hr}$ of intracellular dye injection to the ipsilateral MN5, suggesting that the degree of coupling between the two pairs of neurons had decreased (Fig. $5 J$ ). To examine the course of the $\mathrm{X}$ axon, we took advantage of the fact that the MN5 and X cell bodies become separated at 16-18 hr APF, just before their dye coupling is abolished (Fig. $2 H$ ). Intracellular injection of Lucifer yellow into both neurons revealed that the axon of $\mathrm{X}$ neuron reached the contralateral side of the neuromere by traveling in close apposition to the MN5 axon and then bifurcated to send one branch anteriorly (Fig. $5 K$, inset), with the other branch continuing parallel to the MN5 axon. This branch stopped at the base of the T2 nerve, in contrast to the MN5 axon, which continued in the T2 nerve (Fig. $5 K$, arrow).

In summary, MN5 has an embryonic origin, but its axon terminates proximally within the T2 nerve without innervating larval muscles. It is a developmentally arrested neuron. In the prepupa, the MN5 axon projects into the ISN to innervate the DLM templates. MN5 is coupled to the X neuron, and both are coupled to their contralateral counterparts in the mature larva, prepupa, and early pupa. The axon of the $\mathrm{X}$ neuron is restricted to the CNS at these stages.

mesothoracic (T2) hemisegment of a wandering third instar larva. The mesothoracic nerve was filled anterogradely with biocytin, and the staining was revealed with $\mathrm{Cy} 3$-conjugated Streptavidin ( red $)$. The filamentous actin of the muscles was revealed with Oregon Green-phalloidin ( green). Dotted lines indicate the approximate boundaries between dorsal $(D)$, lateral $(L)$, and ventral $(V)$. 

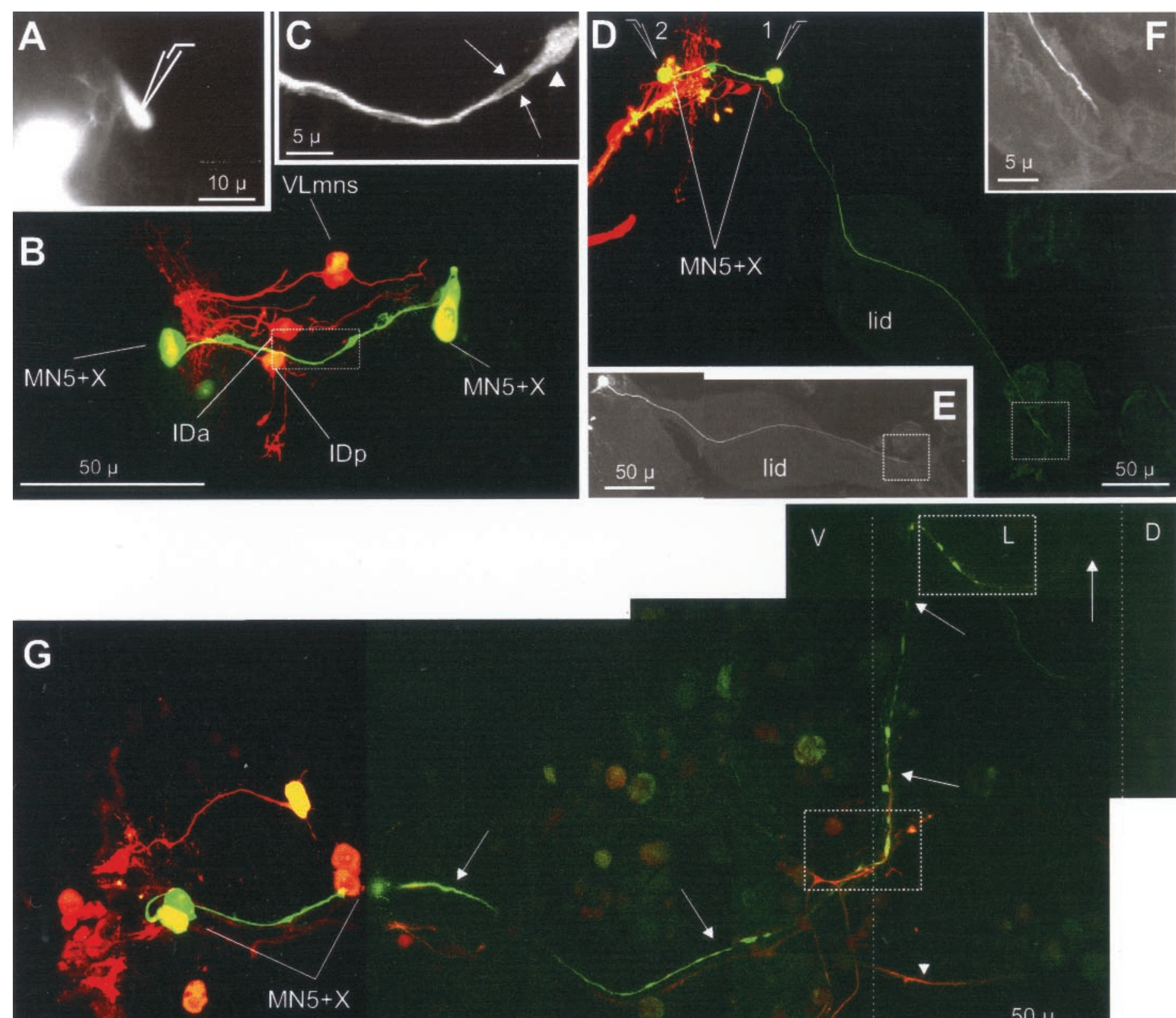

lid

\section{(3)}
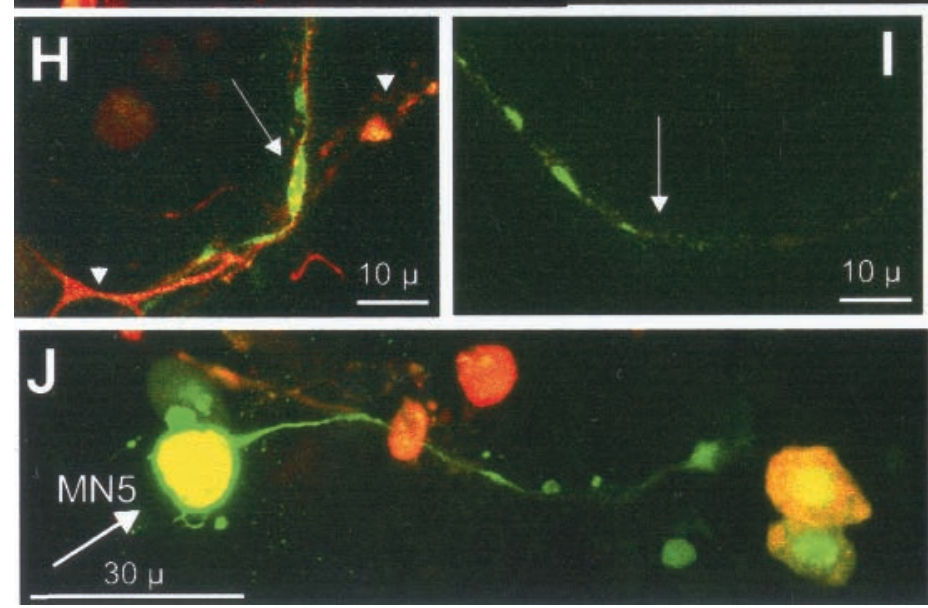
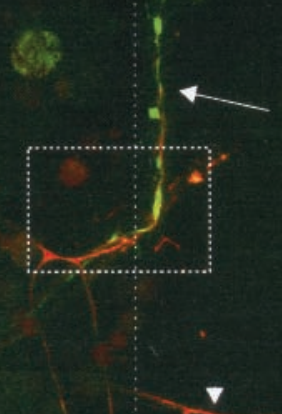

$50 \mu$
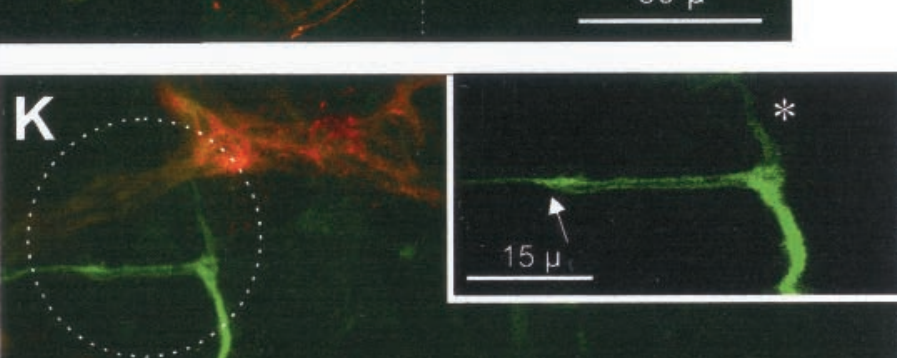

Figure 5. MN5 is a developmentally arrested neuron in the wandering larva and prepupa. All panels, except $A$, are projections of confocal optical sections $(z=0.5 \mu \mathrm{m})$ from Canton-S animals. $A$, Fluorescent image taken from a live larval preparation in saline showing the right pair of MN5 and $\mathrm{X}$ neuron that were stained with $3 \mathrm{kDa}$ rhodamine-dextran after filling the left mesothoracic nerve. B, Confocal image of (Figure legend continues.) 


\section{Dendritic growth of MN5}

Intracellular Lucifer yellow or Neurobiotin injections revealed the dendritic growth of MN5 at high resolution. Before impalement, MN5 was identified in dextran backfills of the T2 nerve, as detailed above, or visualized in transmitted light based on the unique position and size of the cell body. In P103.3/UAS-GFP animals, the $G A L 4$ driver directs GFP reporter gene expression in MN5 after $35 \mathrm{hr}$ APF, allowing the intracellular injection of Neurobiotin under visual control.

MN5 lacks dendrites in larval, prepupal, and early pupal stages. The first primary dendrite becomes apparent by $19 \mathrm{hr}$ APF (Fig. $6 A$, arrow). By 20-22 hr APF, more primary dendrites associated with a halo of very fine processes become apparent (Fig. 6B). Within the next $5 \mathrm{hr}$, additional primary branches are added (Fig. $6 C)$. During the next $15 \mathrm{hr}(25-40 \mathrm{hr}$ APF), the primary dendrites become longer, and finer secondary processes begin to extend from the primary branches (Fig. 6D), whereas by $40 \mathrm{hr}$ APF, an anterior branch has turned and crossed the midline (Fig. 6E, arrow). A second anterior branch and several posterior branches cross the midline during the next $10 \mathrm{hr}$ (Fig. 6F, G). By $75 \mathrm{hr}$ APF, MN5 has acquired most of the features of the adult-like dendritic pattern (Fig. 6H). During the remaining stages of pupal development, dendrites undergo a significant shift in position as the CNS acquires its final shape. Elongation and sprouting of highorder dendritic branches continues until the adult stage (Fig. 6I). In conclusion, primary dendrites emerge from specific points of the neurite shaft and grow mostly perpendicular to the neurite shaft covering a large percentage of the mesothoracic neuromere. Their number and pattern of growth are stereotypic among preparations of the same pupal stage. The cell body of MN5 grows considerably larger between 20 and $30 \mathrm{hr}$ APF (Fig. 7), and its position relative to the axon changes from posterior to anterior (Fig. 6B-D).

\section{DISCUSSION}

\section{Origin and metamorphic fate of MN1-MN5 and $X$ neurons}

The conversion from the larval to the adult CNS involves the degeneration of obsolete larval neurons, the differentiation of new neurons produced by neuroblasts during larval and pupal stages, and the remodeling of persistent larval neurons (for review, see Levine and Weeks, 1990; Weeks and Levine, 1990; Truman et al., 1993; Levine et al., 1995; Consoulas et al., 2000;
Tissot and Stocker, 2000). Persistent motoneurons undergo profound changes in dendritic morphology and synaptic interactions during metamorphosis, reflecting behavioral requirements of the adult. In moths, adult motoneurons, including the homologous MN1-MN5 neurons of Manduca (Casaday and Camhi, 1976; Duch and Levine, 2000; Duch et al., 2000) and silk moth Bombyx mori (Tsujimura, 1988, 1989), are derived from larval motoneurons that have survived metamorphosis. It has been assumed that the adult Drosophila MN1-MN5 are persistent, remodeled larval motoneurons (Hummon and Costello, 1987; Fernandes and Vijayraghavan, 1993; Truman et al., 1993, 1994; Fernandes and Keshishian, 1998; Tissot and Stocker, 2000), but definitive evidence has been lacking until this study.

Like some Kenyon cells of the mushroom bodies (Technau and Heisenberg, 1982; Lee et al., 1999) and some pharyngeal muscle motoneurons in Drosophila (Tissot et al., 1998), MN1-MN4 are born embryonically and persist, undergoing metamorphic remodeling (for a summary of the events, see Fig. 8). MN5 represents a novel class of motoneurons that are developmentally arrested in the larva but finish differentiating and become functional during metamorphosis (Fig. 8). The Drosophila tergotrochanteral motoneuron (TTMn) (Jacobs et al., 2000) and leg motoneurons [C. M. Bate, unpublished data (cited in Truman et al., 1993); C. Consoulas, unpublished observations] (Truman et al., 1993) may have similar metamorphic histories.

These data provide insight into the development of a neural circuit. In adult flies, a stereotyped escape response, consisting of a jump and initiation of flight, is mediated by the giant fiber (GF) system (Thomas and Wyman, 1983). This is the best described circuit in Drosophila and readily amenable to genetic and electrophysiological approaches (Tanouye and Wyman, 1980; Thomas and Wyman, 1983; Trimarchi and Schneiderman, 1994b; Phelan et al., 1996; Trimarchi and Murphey, 1997; Allen et al., 1999, 2000; Jacobs et al., 2000). A pair of brain interneurons (GFs), which receive inputs from the eyes and wind-sensitive hairs, project their axons to the mesothoracic neuromere. The GF neurons synapse directly with the TTMn and indirectly via the peripherally synapsing interneuron (PSI) in the PDMN, with MN1-MN5 (Power, 1948; King and Wyman, 1980; Tanouye and Wyman, 1980; Koto et al., 1981; Thomas and Wyman, 1983; Sun and Wyman, 1997).

The GF neurons are born during embryogenesis but delay axogenesis and dendritic development until the end of the larval

\footnotetext{
(Figure legend continued.) the same preparation showing dorsal cell bodies and dendrites of the neurons stained after filling the left mesothoracic nerve with $3 \mathrm{kDa}$ rhodamine-dextran (red) and the pattern of staining after intracellular injection of Lucifer yellow ( green) into the superficial neuron in $A$ (see electrode diagram in $A$ ). The dye had diffused within a few seconds to the right X neuron and the left pair of MN5 and X neuron, demonstrating their coupling. $C$, Higher magnification of the area indicated in the box in $B$. Only two axons can be distinguished (arrows). Note the swelling that may serve as contact points between MN5 and X neurons (arrowhead) (see Results). D, Wandering third instar larva. As in $C$, the right MN5 and X neurons were stained with $3 \mathrm{kDa}$ rhodamine-dextran after filling the left mesothoracic nerve. The two pairs of MN5 and X neurons were stained with Lucifer yellow after intracellular injection of the dye into the right MN5 (electrode 1). The left MN5 was then impaled and stained intracellularly with Lucifer yellow for $45 \mathrm{~min}$ (electrode 2), allowing the dye to diff use into its entire intact axon. $E$, The same preparation as in $D$, in a different orientation to better reveal the left MN5 axon in the right T2 nerve. The axon of the left MN5 exits the CNS and stops in the T2 nerve at a level close to the mesothoracic leg imaginal disc (lid) just before the nerve branches into ISN and SN. $F$, Higher magnification of the boxed areas in $D$ and $E$. $G$, Prepupa, 4 hr APF The left T2 nerve was filled with $3 \mathrm{kDa}$ rhodamine-dextran $(\mathrm{red})$ to reveal the two pairs of MN5 and X neuron. The left MN5 was injected intracellularly with Lucifer yellow ( green) for $45 \mathrm{~min}$, revealing the course of its intact axon (arrows) in the right mesothoracic hemisegment along the ventral ( $V$ ) and lateral $(L)$ body wall. The axon had almost reached the dorsal $(D)$ thoracic body wall region. $H$, I, Higher-magnification images of the boxed areas indicated in $G$. Note the peripheral processes of VUM neurons that were stained with $3 \mathrm{kDa}$ rhodamine-dextran attributable to the retrograde nerve fill (arrowheads). J, Prepupa, $8 \mathrm{hr}$ APF. The nature of the coupling among the four cells (MN5 and X) had changed. In contrast to the larva, 1 hr of Lucifer yellow injection into the MN5 (arrow) was required to stain the other three cells in the prepupa. K, Pupa, 16 hr APF. The right MN5 and X neuron were labeled after backfilling the left T2 nerve with $3 \mathrm{kDa}$ rhodamine-dextran. Both cells were then stained intracellularly with Lucifer yellow. Note that the axon of the $\mathrm{X}$ neuron bifurcates into two branches (encircled area). Inset shows the encircled area in $K$ at a higher magnification. In contrast to the axon of MN5, the axon of X neuron stops at the base of the T2 nerve (arrow) after giving rise to an anterior branch (asterisk).
} 

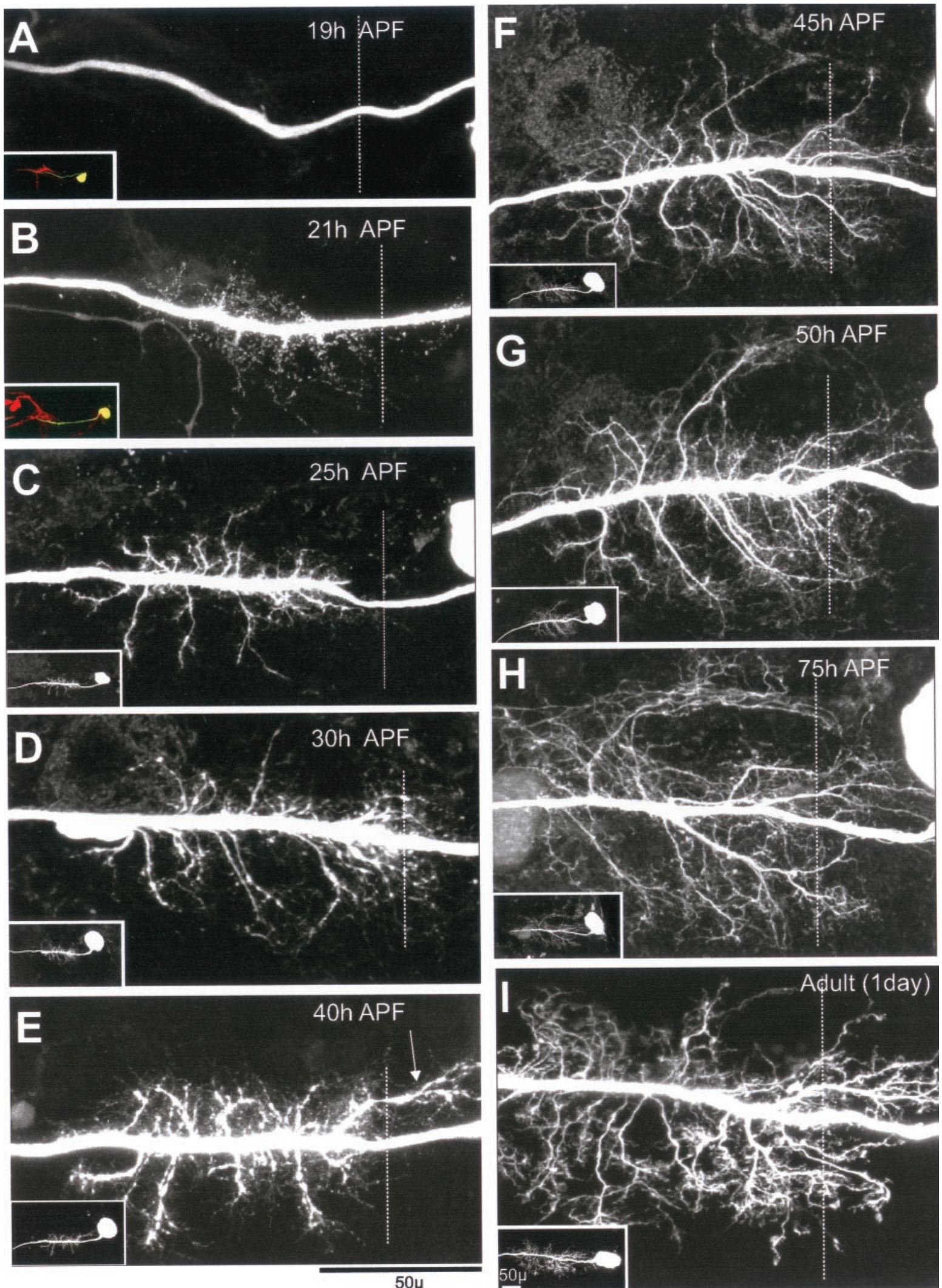

Figure 6. De novo dendritic outgrowth of MN5. Projections of confocal optical sections $(z=0.5 \mu \mathrm{m})$ of MN5 dendrites taken from representative preparations of sequential stages between the early pupa (19 hr APF) and the adult. Insets are confocal images of the same preparations at a lower magnification to include the cell bodies. In $A$ and $B$, the cell body of MN5 was identified by backfilling its axon in Canton-S animals with 3 kDa rhodamine-dextran (red). Subsequently, MN5 was intracellularly injected with Lucifer yellow ( green). In $C$ and $D$, the MN5 cell body of Canton-S animals was visually identified using DIC optics. In $E-I$, GFP expression in MN5 of P103.3/UAS-GFP animals facilitated its identification. MN5 was intracellularly injected with Neurobiotin, and the staining was revealed with Cy3-conjugated Streptavidin. Anterior is to the top. Dotted lines indicate midline. 

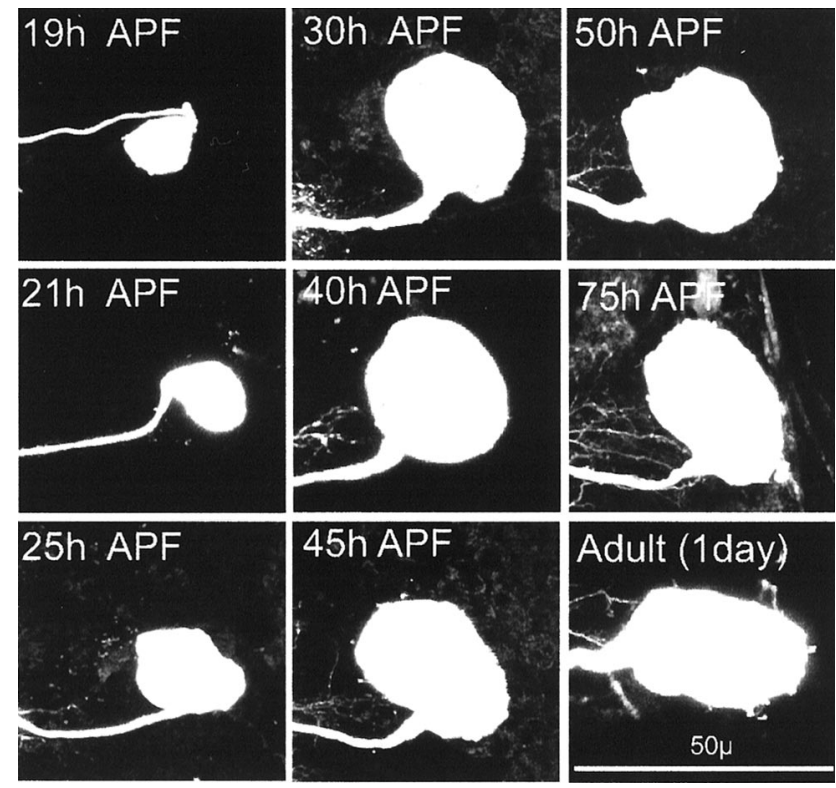

Figure 7. MN5 cell body increases in size during metamorphosis. The cell bodies of intracellularly stained MN5, whose dendrites are shown in Figure 5. Note the dramatic increase in size of the MN5 cell body.

life (Allen et al., 1998). In the middle of the pupal stage (later than $28 \mathrm{hr}$ APF), the PSI is dye coupled to the TTMn and to the GF (Phelan et al., 1996; Jacobs et al., 2000). Based on the size and position of the cell body and the course of its primary neurite (see below), we propose that the $\mathrm{X}$ interneuron is the PSI. Thus, it is likely that all of the neurons in this escape circuit are born in the embryo but differentiate for their adult function during the metamorphic transition.

The PSI neurite must travel through the mesothoracic neuromere, cross the midline, enter the PDMN, and stop at a specific point within the PDMN. The PSI neurite becomes encircled by the MN1-MN5 axons with which it makes synaptic contacts (Egger et al., 1997). PSI may use MN5 as a pathfinding guide to reach the appropriate locale in the contralateral PDMN. In support of this hypothesis, MN5 and X neurites follow the same trajectory within the CNS at $16 \mathrm{hr} \mathrm{APF}$. The $\mathrm{X}$ neuron is transiently coupled to its contralateral counterpart and to both MN5s in the early phase of metamorphosis (wandering larva to $16 \mathrm{hr}$ APF). In the wandering larva, the contact point between the $X$ and MN5 neurites is close to their cell bodies. By $16 \mathrm{hr}$ APF, the tip of the $\mathrm{X}$ neurite contacts the MN5 axon at a point close to the exit of the mesothoracic nerve. We hypothesize that, during the remaining time of pupal development, the X-MN5 contact point advances into the PDMN. Evidence in favor of this model comes from temperature-sensitive shibire (shi) mutants, in which a heat pulse at $12 \mathrm{hr}$ APF results in developmental arrest of PSI neurite extension. The PSI neurite never reaches its normal position in the PDMN but can be found in the adult mutant flies within the CNS just before the exit of the nerve (Hummon and Costello, 1987), which is where we find the $X$ neuron axon in early pupae.

\section{Time course of dendritic and axonal remodeling}

Although both the dendrites and axon terminals of the flight motoneurons are modified during metamorphosis, the central and peripheral remodeling of MN1-MN5 in Drosophila are not synchronized (Fig. 8). Motoneurons MN1-MN4, IDa, and IDp, undergo dendritic regression very rapidly during an $\sim 2$-hr interval in the early prepupa (2-4 hr APF), followed by a phase of stability (4-19 hr APF). In the periphery, the prepupal DLM template muscles enter a phase of partial degeneration (6-10 hr APF) (Fernandes et al., 1991), and their associated MN1-MN4 motor branches begin to retract (Fernandes and Vijayraghavan, 1993). Thus, dendritic regression (2-4 hr APF) precedes the retraction of the axon terminals of MN1-MN4 from the DLM template fibers (6-10 hr APF) (Fig. 8). Dendritic outgrowth
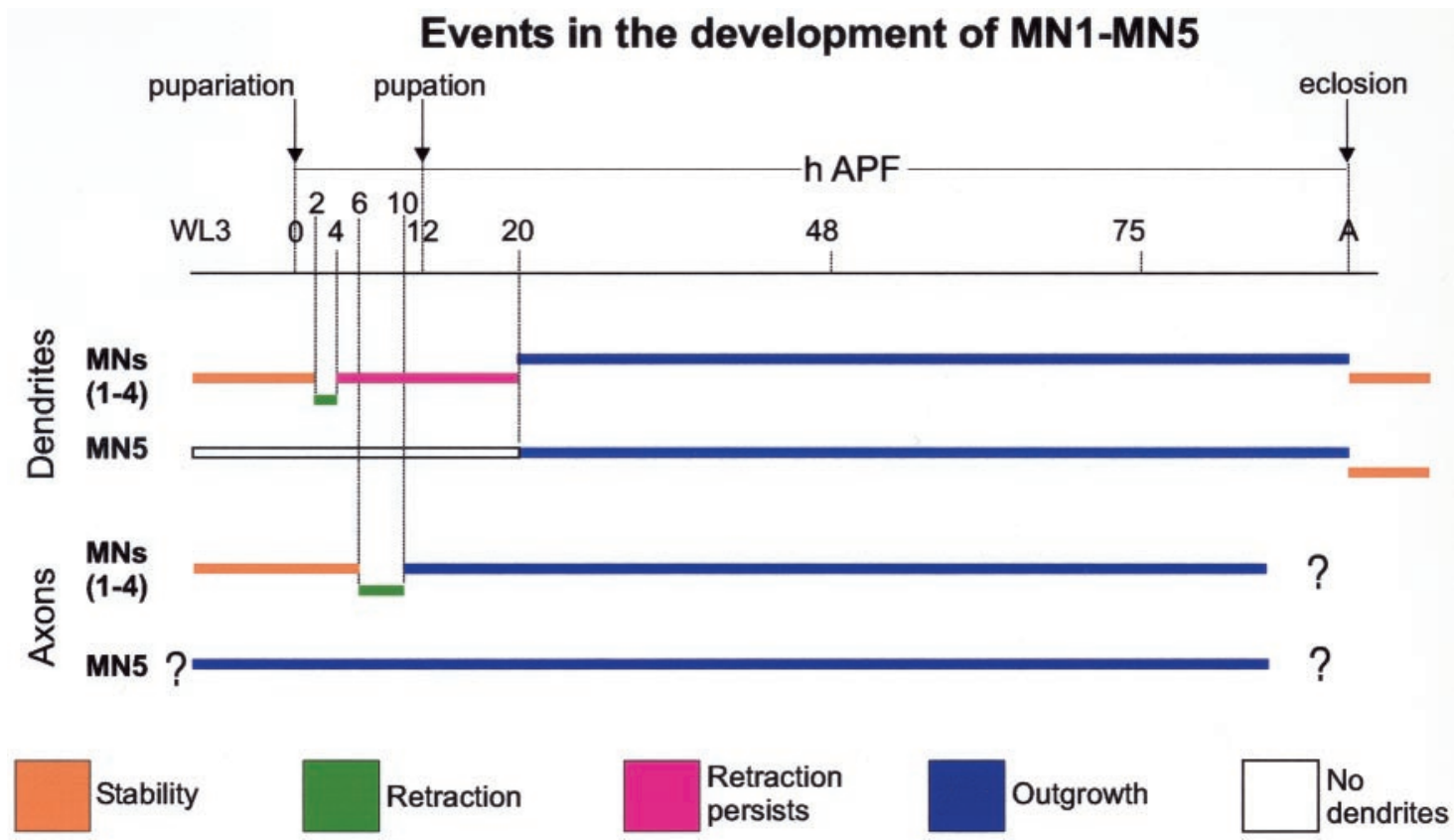

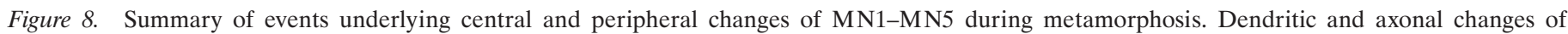

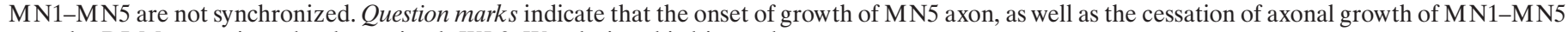
over the DLMs, remain to be determined. WL3, Wandering third instar larva. 
begins simultaneously for MN1-MN4 and MN5 at 19-20 hr APF, whereas the reexpansion of their motor branches over the developing DLMs begins earlier, by $10 \mathrm{hr}$ APF (Fernandes and Vijayraghavan, 1993) (Fig. 8).

\section{Regulation of dendritic growth}

The primary dendrites of MN5 extend perpendicularly from specific regions of the neurite in anterior or posterior directions. As the primary branches grow in length, secondary branches begin to form from their tips and shafts. Lengthening of all primary branches, addition of high-order branches, and bending of some anterior and posterior branches across the midline toward the cell body shape the final pattern of the dendritic arbor of the MN5. Thus, the formation of MN5 dendritic tree occurs in discrete but often overlapping steps. The mechanisms controlling each of these processes may now be examined against this detailed description of normal growth.

Insect steroid hormones, the ecdysteroids, are major regulators of dendritic remodeling. Manipulations of the ecdysteroid titer demonstrate that both dendritic regression and regrowth of persistent Manduca motoneurons are under hormonal control (Weeks and Truman, 1985; Weeks, 1987; Truman and Reiss, 1988; Weeks and Ernst-Utzschneider, 1989; Levine and Weeks, 1990, 1996; Weeks et al., 1992; Streichert and Weeks, 1995). Ecdysteroids act directly on neurons to regulate growth and branching in primary cell culture (Prugh et al., 1992; Levine and Weeks, 1996; Kraft et al., 1998) through regulation of the growth cone cytoskeleton (Matheson and Levine, 1999). Among the genes directly induced by $20 \mathrm{E}$ (primary response genes) is a set of loci encoding transcription factors (Andres and Thummel, 1992), at least one of which, Broad Complex $(B R C)$, is required for metamorphic reorganization of the CNS (Restifo and White, 1991; Liu and Restifo, 1997). One of the transcription factors encoded by $B R C$ is essential for dendritic outgrowth of MN1MN5 during pupal development (our unpublished observations).

In addition to steroid hormones, other extracellular signals (Tessier-Lavigne and Goodman, 1996; Scott and Luo, 2001), synaptic partners, spontaneous and synaptic activity (Cline, 2001), and intrinsic genetic programs (Gao et al., 1999) regulate dendritic growth and guidance. A basic understanding of normal neural function and of how neurons respond to behavioral changes, injury, and disease requires knowledge of both the mechanisms that allow neurons to maintain stable dendritic structures and the signals and transduction pathways that lead to modifications. Linking intrinsic regulators with extrinsic signals and neural activity is a significant challenge (McAllister, 2000; Redmond and Ghosh, 2001). One approach to this challenge is to develop model systems in which regulatory molecules can be manipulated and the consequences assessed by examining the dendritic morphology of identified neurons. MN5 offers a central neuron whose dendritic growth regulation can be analyzed at high resolution through mutation or directed alterations in gene expression.

\section{REFERENCES}

Allen MJ, Drummond JA, Moffat KG (1998) Development of the giant fiber neuron of Drosophila melanogaster. J Comp Neurol 397:519-531. Allen MJ, Shan X, Caruccio P, Froggett SJ, Moffat KG, Murphey RK (1999) Targeted expression of truncated Glued disrupts giant fiber synapse formation in Drosophila. J Neurosci 19:9374-9380.

Allen MJ, Shan X, Murphey RK (2000) A role of Drosophila Drac1 in neurite outgrowth and synaptogenesis in the giant fiber system. Mol Cell Neurosci 16:754-765.

Andres AJ, Thummel CS (1992) Hormones, puffs and flies: the molecular control of metamorphosis by ecdysone. Trends Genet 8:132-138.
Bainbridge SP, Bownes M (1981) Staging the metamorphosis of Drosophila melanogaster. J Embryol Exp Morphol 66:57-80.

Bottjer SW, Arnold AP (1997) Developmental plasticity in neural circuits for a learned behavior. Annu Rev Neurosci 20:459-481.

Brand AH, Perrimon N (1993) Targeted gene expression as a means of altering cell fates and generating dominant phenotypes. Development 118:401-415.

Cameron WE, Nuñez-Abades PA (2000) Physiological changes accompanying anatomical remodeling of mammalian motoneurons during postnatal development. Brain Res Bull 53:523-527.

Casaday GB, Camhi JM (1976) Metamorphosis of flight motor neurons in the moth Manduca sexta. J Comp Physiol 112:143-158.

Cline HT (2001) Dendritic arbor development and synaptogenesis. Curr Opin Neurobiol 11:118-126.

Coggshall JC (1978) Neurons associated with the dorsal longitudinal flight muscle of Drosophila melanogaster. J Comp Neurol 177:707-720.

Consoulas C, Levine RB (1997) Accumulation and proliferation of adult leg muscle precursors in Manduca are dependent on innervation. J Neurobiol 32:531-553.

Consoulas C, Levine RB (1998) Presynaptic function during muscle remodeling in insect metamorphosis. J Neurosci 18:5817-5831.

Consoulas C, Kent KS, Levine RB (1996) Remodeling of the peripheral processes and presynaptic terminals of leg motoneurons during metamorphosis of the hawkmoth, Manduca sexta. J Comp Neurol 372:415-434.

Consoulas C, Duch C, Bayline RJ, Levine RB (2000) Behavioral transformations during metamorphosis: remodeling of neural and motor systems. Brain Res Bull 53:571-583.

Costello WJ, Wyman RJ (1986) Development of an indirect flight muscle in a muscle-specific mutant of Drosophila melanogaster. Dev Biol 118:247-258.

Cubitt AB, Heimar R, Adams SR, Boyd AE, Gross LA, Tsien RY (1995) Understanding, improving and using green fluorescent proteins. Trends Biochem Sci 20:448-455.

Duch C, Levine RB (2000) Remodeling of membrane properties and dendritic architecture accompanies the postembryonic conversion of a slow into a fast motoneuron. J Neurosci 20:6950-6961.

Duch C, Bayline RJ, Levine RB (2000) Postembryonic development of the dorsal longitudinal flight muscle and its innervation in Manduca sexta. J Comp Neurol 422:1-17.

Egger MD, Nowakowski RS, Peng B, Wyman RJ (1997) Patterns of connectivity in a Drosophila nerve. J Comp Neurol 387:63-72.

Elgin SR, Miller DW (1978) Mass rearing of flies and mass production and harvesting of eggs. In: The genetics and biology of Drosophila (Ashburner M, Wright TRF, eds), Vol 2A, pp 112-121. New York: Academic.

Fernandes J, Vijayraghavan K (1993) The development of indirect flight muscle innervation in Drosophila melanogaster. Development 118:215-227.

Fernandes JJ, Keshishian H (1998) Nerve-muscle interactions during flight muscle development in Drosophila. Development 125:1769-1779.

Fernandes J, Bate M, Vijayraghavan K (1991) Development of indirect flight muscles of Drosophila. Development 113:67-77.

Gao FB, Brenman JE, Jan LY, Jan YN (1999) Genes regulating dendritic outgrowth, branching, and routing in Drosophila. Genes Dev 13:2549-2561.

Hummon MR, Costello WJ (1987) Induced disruption in the connectivity of an identified neuron in the Drosophila ts mutant shibire. J Neurosci 7:3633-3638.

Ikeda K, Koenig JH (1988) Morphological identification of the motor neurons innervating the dorsal longitudinal flight-muscle of Drosophila melanogaster. J Comp Neurol 273:436-444.

Jacobs K, Todman MG, Allen MJ, Davies JA, Bacon JP (2000) Synaptogenesis in the giant-fibre system of Drosophila: interaction of the giant fibre and its major motoneuronal target. Development 127:5203-5212.

Kent KS, Levine RB (1993) Dendritic reorganization of an identified neuron during metamorphosis of the moth, Manduca sexta: the influence of interactions with the periphery. J Neurobiol 24:1-22.

King DG, Wyman RJ (1980) Anatomy of the giant fiber pathway in Drosophila. I. Three thoracic components of the pathway. J Neurocytol 9:753-770.

Koto M, Tanouye MA, Ferrus A, Thomas JB, Wyman RJ (1981) The morphology of the cervical giant fiber neuron of Drosophila. Brain Res 221:213-217.

Kraft R, Levine RB, Restifo LL (1998) The steroid hormone 20hydroxyecdysone enhances neurite growth of Drosophila mushroom body neurons isolated during metamorphosis. J Neurosci 18:8886-8899.

Lee T, Lee A, Luo L (1999) Development of the Drosophila mushroom bodies: sequential generation of three distinct types of neurons from a neuroblast. Development 126:4065-4076.

Levine RB, Truman JW (1982) Metamorphosis of the insect nervous system: changes in the morphology and synaptic interactions of identified cells. Nature 299:250-252.

Levine RB, Truman JW (1985) Dendritic reorganization of abdominal 
motoneurons during metamorphosis of the moth, Manduca sexta. J Neurosci 5:2424-2431.

Levine RB, Weeks JC (1990) Hormonally mediated changes in simple reflex circuits during metamorphosis in the moth, Manduca sexta. J Neurobiol 21:1022-1036.

Levine RB, Weeks JC (1996) Cell culture approaches to understanding the actions of steroid hormones on the insect nervous system. Dev Neurosci 18:73-86.

Levine RB, Morton DB, Restifo LL (1995) Remodeling of the insect nervous system. Curr Opin Neurobiol 5:28-35.

Liu E, Restifo LL (1997) Identification of a broad complex-regulated enchancer in the developing visual system of Drosophila. J Neurobiol 34:253-270.

Matheson SF, Levine RB (1999) Steroid hormone enhancement of neurite outgrowth in identified insect motoneurons involves specific effects on growth cone form and function. J Neurobiol 38:27-45.

McAllister AK (2000) Cellular and molecular mechanisms of dendritic growth. Cereb Cortex 10:963-973.

McEwen BS (1999) Stress and hippocampal plasticity. Annu Rev Neurosci 22:105-122.

Phelan P, Nakagawa M, Wilkin MB, Moffat KG, O'Kane CJ, Davies JA, Bacon JP (1996) Mutations in shaking-B prevent electrical synapse formation in the Drosophila giant fiber system. J Neurosci 16:1101-1113.

Poirazi P, Mel BW (2001) Impact of active dendrites and structural plasticity on the memory capacity of neural tissue. Neuron 29:776-796.

Power ME (1948) The thoracico-abdominal nervous system of an adult insect, Drosophila melanogaster. J Comp Neurol 88:347-409.

Prugh JK, Della Croce K, Levine RB (1992) Effects of the steroid hormone, 20-hydroxyecdysone, on the growth of neurites by identified insect motoneurons in vitro. Dev Biol 154:331-347.

Redmond L, Ghosh A (2001) The role of Notch and Rho GTPase signaling in the control of dendritic development. Curr Opin Neurobiol 11:111-117.

Restifo LL, Hauglum W (1998) Parallel molecular genetic pathways operate during CNS metamorphosis in Drosophila. Mol Cell Neurosci 11:134-148.

Restifo LL, White K (1991) Mutations in a steroid hormone-regulated gene disrupt the metamorphosis of the central nervous system in Drosophila. Dev Biol 148:174-194.

Restifo LL, Estes PS, DelloRusso C (1995) Genetics of ecdysteroidregulated central nervous system metamorphosis in Drosophila. Eur J Entomol 92:169-187.

Scott EK, Luo L (2001) How do dendrites take their shape? Nat Neurosci 4:359-365.

Shatoury HE (1956) Developmental interactions in the development of the imaginal muscles of Drosophila. J Embryol Exp Morphol 4:228239.

Stewart BA, Atwood HL, Renger JJ, Wang J, Wu CF (1994) Improved stability of Drosophila larval neuromuscular preparations in hemolymph-like physiological solutions. J Comp Physiol [A] 175:179-191.

Streichert LC, Weeks JC (1995) Decreased monosynaptic sensory input to an identified motoneuron is associated with steroid-mediated dendritic regression during metamorphosis in Manduca sexta. J Neurosci 15:1484-1495

Sun YA, Wyman RJ (1997) Neurons of the Drosophila giant fiber system. I. Dorsal longitudinal motor neurons. J Comp Neurol 387:157-166.

Swann JW, Al-Noori S, Jiang M, Lee CL (2000) Spine loss and other dendritic abnormalities in epilepsy. Hippocampus 10:617-625.

Tanouye MA, Wyman RJ (1980) Motor outputs of the giant nerve fiber in Drosophila. J Neurophysiol 44:405-421.

Technau G, Heisenberg M (1982) Neural reorganization during meta- morphosis of the corpora pedunculata in Drosophila melanogaster. Nature 295:405-407.

Tessier-Lavigne M, Goodman CS (1996) The molecular biology of axon guidance. Science 274:1123-1133.

Thomas JB, Wyman RJ (1983) Normal and mutant connectivity between identified neurons in Drosophila. Trends Neurosci 6:214-219.

Thorn RS, Truman JW (1989) Sex-specific neuronal respecification during the metamorphosis of the genital segments of the tobacco hornworm moth Manduca sexta. J Comp Neurol 284:489-509.

Tissot M, Stocker RF (2000) Metamorphosis in Drosophila and other insects: the fate of neurons throughout the stages. Prog Neurobiol 62:89-111.

Tissot M, Gendre N, Stocker RF (1998) Drosophila P[GAL4] lines reveal that motor neurons involved in feeding persist through metamorphosis. J Neurobiol 37:237-250.

Trimarchi JR, Murphey RK (1997) The shaking- $B^{2}$ mutation disrupts electrical synapses in a flight circuit in adult Drosophila. J Neurosci 17:4700-4710.

Trimarchi JR, Schneiderman AM (1994a) The motor neurons innervating the direct flight muscles of Drosophila melanogaster are morphologically specialized. J Comp Neurol 340:427-443.

Trimarchi JR, Schneiderman AM (1994b) Different neural pathways coordinate Drosophila flight initiations evoked by visual and olfactory stimuli. J Exp Biol 198:1099-1104.

Truman JW, Bate M (1988) Spatial and temporal patterns of neurogenesis in the central nervous system of Drosophila melanogaster. Dev Biol 125:145-157.

Truman JW, Reiss SE (1988) Hormonal regulation of the shape of identified motoneurons in the moth, Manduca sexta. J Neurosci 8:765-775.

Truman JW, Taylor BJ, Awad T (1993) Formation of the adult nervous system. In: The development of Drosophila melanogaster (Bate $\mathrm{M}$ Martinez-Arias A, eds), pp 1245-1275. Cold Spring Harbor, NY: Cold Spring Harbor Laboratory.

Truman JW, Talbot WS, Fahrbach SE, Hogness DS (1994) Ecdysone receptor expression in the CNS correlates with stage-specific responses to ecdysteroids during Drosophila and Manduca development. Development 120:219-234.

Tsujimura H (1988) Metamorphosis of wing motor system in the silk moth, Bombyx mori L. (Lepidoptera: Bombycidae): anatomy of the sensory and motor neurons that innervate larval mesothoracic dorsal musculature, stretch receptors, and epidermis. Int J Insect Morphol Embryol 17:367-380.

Tsujimura H (1989) Metamorphosis of wing motor system in the silk moth, Bombyx mori: origin of wing motor neurons. Dev Growth Differ 31:331-339.

Weeks JC (1987) Time course of hormonal independence for developmental events in neurons and other cell types during insect metamorphosis. Dev Biol 124:163-176.

Weeks JC, Ernst-Utzschneider K (1989) Respecification of larval proleg motoneurons during metamorphosis of the tobacco hornworm, Manduca sexta. Segmental dependence and hormonal regulation. J Neurobiol 20:569-592.

Weeks JC, Levine RB (1990) Postembryonic neuronal plasticity and its hormonal control during insect metamorphosis. Annu Rev Neurosci 13:183-194.

Weeks JC, Truman JW (1985) Independent steroid control of the fates of motoneurons and their muscles during insect metamorphosis. J Neurosci 5:2290-2300.

Weeks JC, Davidson SK, Debu HG (1992) Effects of a protein synthesis inhibitor on the hormonally mediated regression and death of motoneurons in the tobacco hornworm, Manduca sexta. J Neurobiol 24:125-140.

Woolley CS (1998) Estrogen-mediated structural and functional synaptic plasticity in the female rat hippocampus. Horm Behav 34:140-148 